\title{
IMPROVED IDENTIFICATION OF IRIS AND EYELASH FEATURES
}

\author{
Richard Youmaran, L.P. Xie and Andy Adler \\ Carleton University, Ottawa, Ontario \\ adler@sce.carleton.ca
}

\begin{abstract}
This paper proposes a novel algorithm to improve localization and segmentation of an iris image. Since in many practical applications, user cooperation is not possible, eyelash occlusion can seriously affect the performance of an iris recognition system. This paper discusses a robust method for accurate localization and segmentation of the exact iris region without eyelash occlusion. Our algorithm uses a logarithmic image enhancement technique and the Hough transform for iris localization as well as an intensity gradient based method for eyelash detection using local region statistics of the image. Experimental results show the accuracy of our algorithm leading to exact iris region segmentation.
\end{abstract}

\section{INTRODUCTION}

Proper Iris segmentation is essential for various security applications using iris recognition technology for personal identification [1]. Irises are occluded by the eyelid and eyelashes as well as from specular reflections from the (typically infra-red) illumination system. In order to accurately process the image, it is important to identify such occluded regions to remove them from further processing. Inaccurate detection of these occlusions reduces considerably the performance of an iris-based identification system when subject cooperation is not possible. Cooperative users can be asked to stand still for multiple image acquisitions, while for Iris On the Move [11] or covert surveillance applications (i.e. in airport security) such cooperation is not available. This will greatly affect the localization of the iris inner and outer boundaries as well as it will degrade the iris feature extraction process. For this reason, exact eyelash detection and segmentation is required to improve the entire biometrics system's accuracy and avoiding poor recognition performance.

In this paper we develop an algorithm for accurate iris segmentation in images where the major portion of the iris is occluded. Our algorithm detects separable and multiple eyelashes, respectively. Separable eyelashes are first detected using a local intensity variation based algorithm while multiple eyelashes are found using the block mean and variance approach. Various methods have been proposed for eyelash detection $[2,3,4,5]$ which uses 1-D Gabor filter, intensity variance, phase congruency, template mean, standard deviation for multiple eyelash detection and a local intensity minimum method for separable eyelash detection. All these methods perform generally quite well but do suffer from some limitations such as computational complexity, inexact iris boundaries segmentation, false eyelash detection and improper eyelash segmentation over the iris region. Specifically, all previous approaches tend to overestimate the occluded regions, and thus lose iris information that could be used for identification. This information loss is potentially important in the covert surveillance applications we consider in this paper. For this reason, the proposed method addresses all these issues using a collection of image processing techniques such as: logarithmic (i.e. non-linear) image enhancement, edge detection, morphological operators, Hough transform, intensity gradient based algorithm and a block mean and variance method using region's local statistics. Our main algorithm is presented in section 2; experimental results are presented in section 3 and a discussion in section 4. Finally, section 5 concludes this paper.

\section{ALGORITHM DESIGN}

This section develops an algorithm to automate the detection and segmentation of eyelash features in an eye image. The design criterion is to determine the detailed eyelash regions without overestimation (falsely detecting iris regions in the image as eyelash). In performing this calculation, it must localize and segment the pupil-iris region using the Hough transform technique $[8,9]$ and a non-linear image enhancement algorithm on the iris region in order to facilitate eyelash detection. All images are of size $480 \times 640$ pixels and are taken under the same sampling and illumination conditions.

\subsection{Pupil-Iris region localization and boundary extraction}

This section describes an algorithm for accurate iris boundary detection and contour extraction (Fig. 1) based on a combination of image processing techniques.

\subsubsection{Non-linear image enhancement}

The original eye image is low pass filtered with a $5 \times 5$ Gaussian filter with $N=4$ iterations (ideal for these images), using the non-linear edge and contrast enhancement algorithm described in $[6,7]$. In order to avoid loss of information, arithmetic operations on image pixel values are defined in a logarithmical mapped space where the forward mapping function between the image pixel space $(F)$ and the real number space $(\psi)$ is $\psi(F)=\log ((255-F) / F)$. The 
iterative technique described overcomes the limitations of linear methods by performing a non-linear weighting operation on the input pixels of the image. This requires the selection of parameters $s_{i}$ to control the amount of high frequency content introduced in the solution. If $s_{i}<1$, the solution will be smoothed otherwise, it amplifies edges. The output of this system results in a binarized enhanced image with sharper edges and better contrast (Fig. 3(c)).

\subsubsection{Edge detection}

Using the binary image, a Sobel operator is constructed to perform a 2-D spatial gradient measurement on an image and gives more emphasis to high-frequency regions that correspond to edges.

The Sobel operator consists of a pair of $3 \times 3$ convolution kernels, which are designed to find lines in an image. The edge map is shown in Fig. 4 (b).

\subsubsection{Hough transform}

The Hough transform is a technique which can be used to isolate features of a particular shape within an image. In this section, it is used to locate the iris outer boundary. The Hough transform is applied directly on an edge map to reduce processing time.

The Hough transform represents an image in terms of a 3dimensional accumulator array. For example, circles correspond to the equation $(x-a)^{2}+(y-b)^{2}=r^{2}$ which defines a circle of center $(a, b)$ and radius $r$ in the $x-y$ space. For this specific feature, the accumulator array will contain the $a, b, r$ parameters which are updated for each edge pixel $(x, y)$. After updating the parameters for all pixels in the edge map, peaks in the accumulator array indicate the location of the desired feature (i.e. circle). From this information, the iris boundary is located (Fig. 4 (c)) and the iris-pupil region is segmented for further processing (section 2.1.4).

\subsubsection{Accurate iris segmentation}

The iris contour given using the Hough transform technique in section 2.1.3 is an approximation of the proper iris boundary which is not always circular. To correct the offset on the iris contour, we recalculate a new contour using the edge map obtained in section 2.1.2. Starting at the center position of the approximated contour, scan outwards for the first set of pixels, different from black, that form a closed contour near the boundary computed in section 2.1.3. This process is shown in (Fig. 4(b)). The exact pupil-iris segmented region is shown in Fig. 4 (d).

\subsection{Eyelash detection}

In this section, separable and multiple eyelashes are detected using an intensity gradient based algorithm and a block mean and variance method (Fig. 2). The iris and non-iris images (Fig. 4(d, f)) are processed independently for improved image enhancement (Fig.4(e, g)) and precise eyelash detection based on the local region statistics and finally, the computed eyelash points are combined for exact iris region extraction.

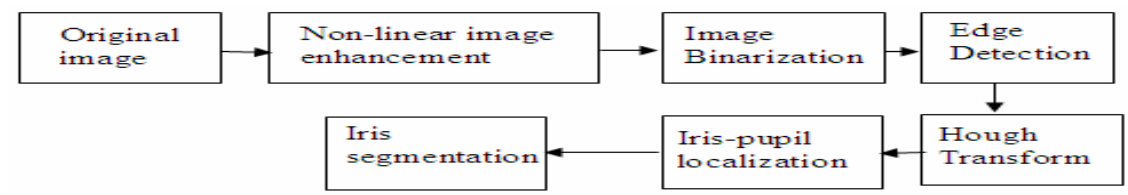

Figure 1: Iris segmentation algorithm based on local image enhancement

A final eyelash map is created by selecting strong

\subsubsection{Local image enhancement}

The iris region (Fig. 4(d)) and the non-iris image (Fig. 4(f)) are enhanced separately using the method described in section 2.1.1. This will improve eyelash detection since it depends on the local image statistics. The iris region tends to contain higher intensity variation than the overall eye image. The enhancement results are shown in Fig. 4(e, g), respectively.

\subsubsection{Separable eyelashes}

In order to detect separable eyelashes in the horizontal, vertical and diagonal direction, the image obtained in section 2.2.1 is convoluted with the developed masks as shown in equation 1. An image with all possible eyelash points is created. A possible eyelash candidate point is set to " 0 " when the mask response $R_{i}$ is negative and to " 1 ", otherwise. eyelash candidates only. This is obtained by taking $R_{i}<T$ where $T$ is a threshold selected to be -200 in our experiment.

$R_{i}(x, y)=\sum_{m=-N}^{N} \sum_{n=-N}^{N} I(x-m, y-n) M(m, n)$

where $I(x, y)$ is the enhanced image, $R_{i}(x, y)$ is the mask response at position $(x, y)$ and $M$ is a $(2 N+1 \times 2 N+1)$ convolution mask. This condition also satisfies the connective criterion [2] where each eyelash point should connect to another point on an eyelash or to an eyelid.

Using the developed masks, a negative mask response is obtained if and only if the center pixel is located between two adjacent eyelash points which satisfies the following connectivity criterion: if the center pixel is surrounded by non-eyelash points, the convolution operation will result in a value greater than the selected threshold which indicates that the pixel is not an eyelash point. 


\subsubsection{Multiple eyelashes}

For regions containing multiple eyelashes, the mean and variance of a $n \times n$ region is taken to detect eyelash candidates. These regions are generally composed of lower intensity pixels with a higher variance. In order to find eyelash candidates in these regions, the computed block mean $u_{\text {bi }}$ (eq. 2) and variance $v_{\text {bi }}$ (eq. 3) are compared to different thresholds. If $u_{\mathrm{bi}}<T_{1}$ or $v_{\mathrm{bi}}>T_{2}$ then the center pixel in block $i$ is considered to be an eyelash point. In our implementation, the block size used is $5 \times 5$.

$$
\begin{aligned}
& \mathrm{u}_{\mathrm{bi}}(x, y)=\frac{1}{n^{2}} \sum_{i=-n}^{n} \sum_{j=-n}^{n} f(x+i, y+j) \\
& \mathrm{v}_{\mathrm{bi}}(x, y)=\frac{1}{n^{2}} \sum_{i=-n}^{n} \sum_{j=-n}^{n}\left(f(x+i, y+j)-\mathrm{u}_{\mathrm{bi}}(x, y)\right)^{2}
\end{aligned}
$$

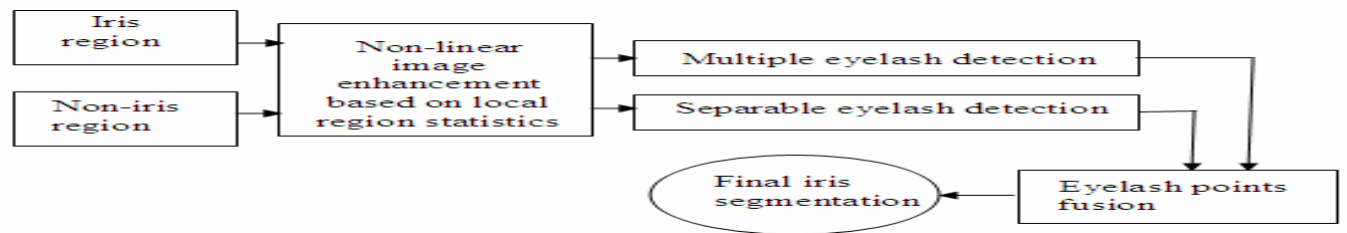

Figure 2: Eyelash detection algorithm and ideal iris region segmentation (link each block to a section in the method

\section{RESULTS}

The algorithm was tested on 327 iris images taken from the CASIA database [10]. Fig. 5(d, e, f) show accurate detection of eyelashes in different images. Fig. $5(\mathrm{~g}, \mathrm{~h}, \mathrm{i})$ illustrates an accurate segmentation of the iris region without eyelash occlusions. The following parameter values were chosen for these results: $s_{1}=0.1, s_{2}=0.1, s_{3}=2, s_{4}=5, s_{5}=150, T_{1}=20$ and $T_{2}$ was set to the variance of the entire region. This process is done for 5 iterations. For the first two iterations, the "s" parameters are set to a value less than one in order to reduce the noise ("smoothing"). Afterwards, the same parameter is set to a value greater than 1 in order to amplify edges assuming that at this stage, noise is inexistent. Experiments were conducted to determine the sensitivity of the results to the parameter choices, and results did not vary significantly for a wide range of parameter choices near the values used.

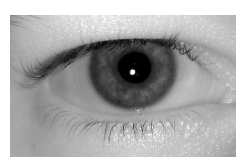

(a)

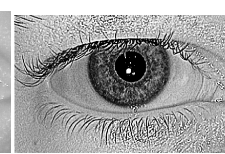

(b)

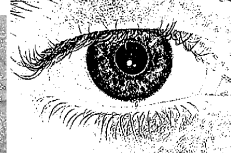

(c)

Figure 3: Image enhancement result: (a) Original image of the eye, (b) Non-linear image enhancement, (c) Binarized image

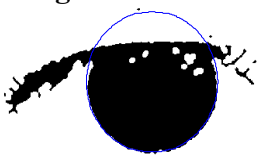

(a)

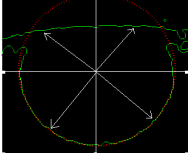

(b)



(c)

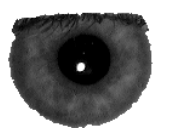

(d)

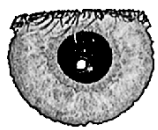

(e)



(f) (g)

Figure 4: Accurate iris boundary extraction and enhancement: (a) Approximated location of the iris outer boundary using the Hough transform, (b) Edge map and accurate iris boundary calculation, (c) Accurate pupil-iris boundary extraction, (d) Exact Pupil-iris region segmentation, (e) Pupil-iris local region enhancement, (f) Non-iris eye image, (g) Non-iris local image enhancement.

(a)

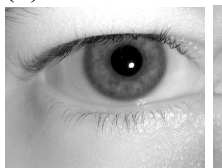

(b)

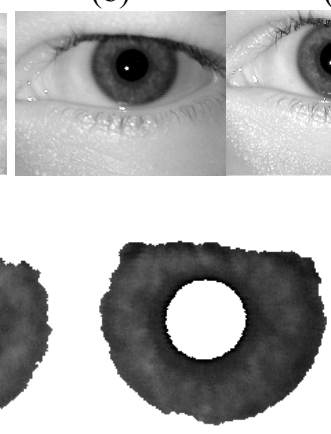

(h) (i)



(c)

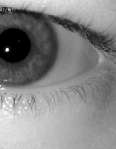

(e)

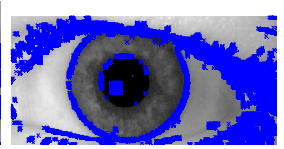

(f)

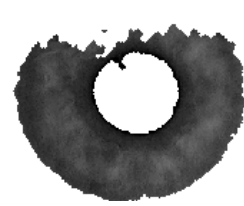

(g)

Figure 5: Eyelash detection and iris segmentation examples: (a, b, c) Original eye images, $(d, e, f)$ Computed candidate eyelash points using our algorithm, $(g, h$, i) Accurate segmentation of the iris regions without eyelash occlusions. 
In addition, we used our technique prior to Daugman's iris recognition system [11] in order to have enhanced eyelash detection before the matching process. This operation will improve detection accuracy and matching performance. Using Daugman's rubber sheet model to represent each iris image in the normalized polar scale, each iris image template is convolved with the Gabor filter to extract the phase feature templates. The phase feature templates are compared to each other using the Hamming distance, and the smallest Hamming distance is used to indicate the correct match. From our testing, it is shown that the identification rate is improved from $93.67 \%$ to $95.25 \%$ as illustrated in the cumulative match curve in Fig. 8. The green curve represents the cumulative match curve without any eyelash detection and the red curve represents the one with eyelash detection. It is clearly shown that the Rank-1 identification rate is improved. Also the DET curve, or FMR-FNMR curve is also improved as in Fig. 6. The top curve represents the performance without eyelash detection and the bottom one represents the performance with eyelash detection. The error rates are improved in almost the entire FMR range.
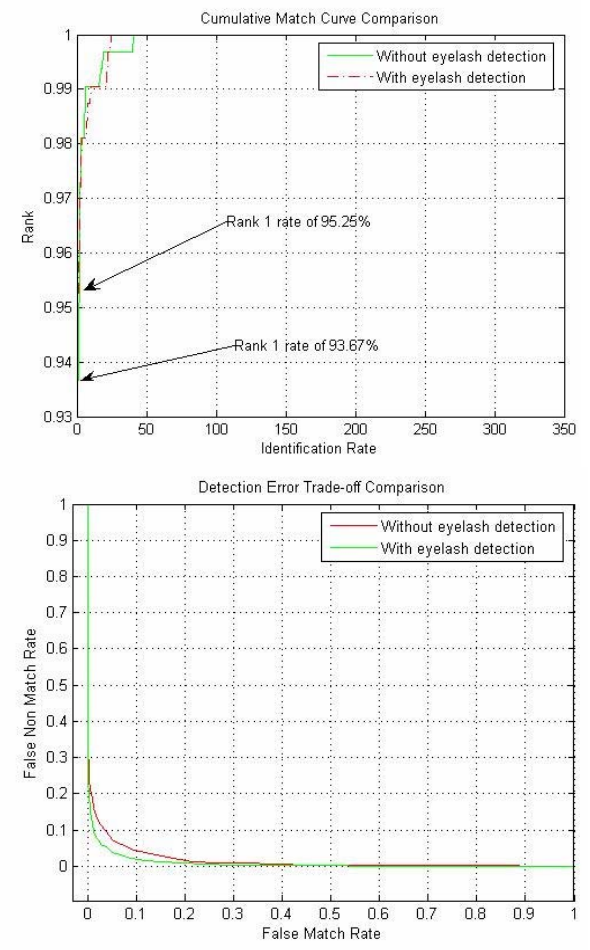

(a)

(b)

Figure 6: (a)Cumulative Match Curve comparison, (b)FMR-FNMR curve comparison

\section{DISCUSSION}

The proposed algorithm shows promising results for eyelash noise detection, accurate iris boundary extraction and ideal iris segmentation. This algorithm locates the iris region using logarithmic image enhancement and the Hough transform techniques, locates the iris boundary, extracts the exact iris contour, detects eyelash based on the local image statistics and block intensity and finally, proposes an ideal iris model for accurate iris recognition. The developed method overcomes the limitations encountered in other iris segmentation and eyelash detection techniques such that our method detects accurately separable and multiple eyelashes, extracts the exact iris contour and is illumination invariant.

\section{CONCLUSION}

This paper proposes a novel eyelash noise detection algorithm that uses a collection of advanced image processing techniques to locate the iris region, extract the exact inner and outer iris boundaries since these are not always circular, detect eyelash noise by combining the eyelash candidate points obtained from separable and multiple eyelash detection techniques based on local image statistics and intensity variance and finally, accurately segment the iris region for further processing.

\section{REFERENCES}

[1] Y. Wang, Y. Zhu and T. Tan, "Personal Identification Based on Iris Recognition", Acta Automatica Sinica, vol. 28, 2002, 1-10.

[2] W.K Kong and D. Zhang, "Accurate Iris Segmentation Based on Novel Reflection and Eyelash Detection Model", Proceedings of International Symposium on Intelligent Multimedia, Video and Speech Processing, Hong Kong, 2001, pp. 263-266.

[3] J. Huang, Y. Wang, T. Tan and J. Cui, "A New Iris Segmentation Method for Recognition", Proceedings of the 17th International Conference on Pattern Recognition, 2004,1051-4651.

[4] X. Yuan and P. Shi, "An Iris Segmentation Procedure for Iris Recognition", Sinobiometrics, 2004, pp. 546-553.

[5] P. Kovesi, "Image Features From Phase Congruency", Journal of Computer Vision Research, vol. 1, no.3, 1999, pp. 1-27.

[6] G. Deng, L.W. Cahill, "Image Enhancement Using the Logratio Approach", Signals Systems and Computers 1, 1994,198-202.

[7] G. Deng, L.W. Cahill, "Multiscale image enhancement using the logarithmic image processing model", Electronics Letters 29, 1993, pp. 803-804.

[8] R.C. Gonzalez, R.E. Woods, Digital Image Processing, Addison-Wesley Publishing Company, 2002.

[9] D.H. Ballard, "Generalizing the Hough transform to detect arbitrary shape", Pattern Recognition, vol. 13:12, 1981,111-122.

[10] Chinese Academy of Sciences Institute of Automation. Casia iris image database. 2004. http://www.sinobiometrics.com

[11] J. Daugman, "How Iris Recognition Works," IEEE Transactions on Systems for Video Technology, vol. 14, no. 1, 2004, pp. 21-30.

[12] Sarnoff Corporation. Iris On The Move, 2005. http://www.sarnoff.com/ 